\title{
Mobile multimedia sensor networks: architecture and routing
}

\author{
Min Chen ${ }^{1}$, Chin-Feng Lai ${ }^{2^{*}}$ and Honggang Wang ${ }^{3}$
}

\begin{abstract}
Recent advances in the fields of wireless technology and multimedia systems have exhibited a strong potential and tendency on improving human life by enabling smart services in ubiquitous computing environments. This paper investigates a mobile multimedia system through combining various technologies, such as wireless sensor networks, embedded multimedia system and node mobility. In particular, we will employ some powerful sensor node with both mobility and multimedia functionalities, which can be controlled by contextual information collected by other systems to enable interactive multimedia services. The new architecture is called mobile multimedia sensor network (MMSN) in this paper. A routing scheme named mobile multimedia geographic routing (MGR) is specially designed to minimize energy consumption and satisfy constraints on the average end-to-end delay of specific applications in MMSNs. Simulations verify the MGR's performance to satisfy QoS requirement while saving energy for MMSNs.
\end{abstract}

Keywords: wireless multimedia sensor networks, multimedia geographic routing, QoS, Internet of Things, energydelay tradeoff, energy efficiency

\section{Introduction}

Recent advances in the fields of wireless technology, multimedia communications [1] and intelligent systems [2] have exhibited a strong potential and tendency on improving human life in every facet, including entertainment, socialization, education, and healthcare. To enable smart multimedia services in a mobile and ubiquitous environment, video surveillance system [3] may interface with other technologies, such as wireless sensor networks (WSNs), wireless multimedia sensor networks (WMSNs) [1,4,5], body area networks [6], Human computer interaction [7], intelligent agent system [8-11], and (cooperative) multi-antenna communication networks [12-15]. With hardware advances, this paper investigates the employment of some powerful sensor node, which is equipped with both mobility and multimedia functionalities, and proposes Mobile Multimedia Sensor Networks (MMSNs). When controlled by contextual information collected by other systems, MMSNs can further support interactive and mobile multimedia services. In this case,

\footnotetext{
* Correspondence: cinfon@ieee.org

${ }^{2}$ Institute of Computer Science and Information Engineering, National Ilan University, llan, Taiwan

Full list of author information is available at the end of the article
}

the marketing opportunities for advanced consumer electronics and services will expand, and more autonomous and intelligent applications will be generated. Yet, various research issues regarding node mobility, coverage, and multimedia streaming over mobile environments are still in clouds for MMSNs.

In this paper, we first present the architecture of MMSNs. Then, we focus on multimedia delivery with the strict quality of service (QoS) requirements. By utilizing location information, we design a routing algorithm with QoS provisioning in an energy-efficient manner. The routing algorithm is called mobile multimedia geographic routing (MGR), which are designed to minimize energy consumption and satisfy constraints on the average end-to-end delay of specific applications while constructing multiple paths to the sink node along the moving trajectory. MGR has the inherent scaling property of geographic routing, where packet-delivery decisions are locally made, and the state at a node is independent of the number of nodes in the network. Most importantly, it achieves flexible energy-delay trade-offs.

Notation used in this paper is given in Table 1. The rest of the paper is organized as follows. Section 2 
Table 1 Notation

\begin{tabular}{ll}
\hline Sym-bol & Definition \\
\hline$s$ & The source node \\
$t$ & The sink node \\
$h$ & The current node \\
$D_{s}^{t}$ & Distance from source node to sink node \\
$D_{h \rightarrow t}$ & Distance from current node to the sink node \\
$T_{\text {hop }}$ & The average hop delay at a sensor node \\
$T_{\text {Qos }}$ & The application-specific end-to-end delay objective \\
$T_{h \rightarrow t}$ & The reserved time credit for the data delivery from current node to the sink node according to $T_{\text {Qos }}$ \\
$t_{s \rightarrow h}$ & Data packet's experienced delay up to current node \\
$H_{h \rightarrow t}$ & The desired hop count from current node to the sink node according to $T_{\text {QoS }}$ \\
$D_{\text {hop }}$ & The desired hop distance for next-hop-selection in MGR \\
$E_{\text {hop }}$ & The energy consumption for one-hop data delivery \\
$E_{\text {ete }}$ & The end-to-end energy consumption for a successful data delivery \\
\hline
\end{tabular}

presents related work. The architecture of MMSNs is presented in Section 3. Section 4 gives an illustrative application for MMSNs. We describe the MGR scheme in Section 5. Simulation model and experiment results are presented in Section 6. Section 7 concludes the paper.

\section{Related work}

Since the proposed MGR is geographic routing scheme for QoS provisioning in mobile multimedia sensor networks, we will introduce the related work in three aspects, i.e., wireless multimedia sensor networks, geographic routing, QoS provisioning for delay sensitive traffic in WSNs.

\subsection{Wireless multimedia sensor networks}

In order to provide reliable and capable high-speed transmission, concurrent multipath routing schemes to enlarge accumulated bandwidth for WMSNs are proposed, such as DGR [4], TPGF [5], Bezier [16], etc. Most of the work focus on how to establish multiple disjointed paths and/or how to control the direction and pattern of the paths. And geographic routing is popular for multipath construction in the existing schemes which assume each intermediate node knows the position information of its neighbors by some positioning techniques.

\subsection{Geographic routing}

Geographic (position-based) routing [17] is a routing scheme in which each sensor node is assumed to be aware of its geographical location, and packet forwarding is performed based on the locations of the nodes. Each node broadcasts a hello message periodically to notify its neighbors of its current position; based on this information, each node sets up a neighbor information table that records the positions of its one-hop neighbors.
In general, each packet is routed to a neighbor closer to the sink than the forwarding node itself until the packet reaches the sink. If a node does not have any neighbors closer to the sink, a fallback mechanism is triggered to overcome this local minimum. Upon arriving at a void, some typical protocols (e.g., GFG [18], GPSR [19], etc.) switch from greedy mode to face mode to circumnavigate the void. When the current node is closer to destination than the node initially starting the face mode, the protocols return to greedy mode (the void is considered circumnavigated) and chooses the next hop using the left/right hand rule.

\subsection{QoS provisioning for delay sensitive traffic in WSNs}

Many applications of WSNs require QoS provisioning for time-constrained traffic, such as real-time target tracking in battlefield environments, emergent event triggering in monitoring applications, etc. Recent years have witnessed increasing research efforts in this area [20-22]. For example, SPEED [23] is an adaptive realtime routing protocol that aims to reduce the end-toend deadline miss ratio in WSNs. MMSPEED [24] extends SPEED to support multiple QoS levels in the timeliness domain by providing multiple packet-delivery delay guarantees. Yuan et al. [25] proposed an integrated energy and QoS aware transmission scheme for WSNs, in which the QoS requirements in the application layer, and the modulation and transmission schemes in the data link and physical layers are jointly optimized. EDDD proposed in [26] provides service differentiation between best-effort and realtime traffic. Our work is closely related to hybrid geographical routing (HGR) [27] scheme which provides a flexible trade-off between energy consumption and end-to-end delay. The HGR scheme is further extended to DHGR (dynamic hybrid geographical routing) to satisfy the end-to-end average packet delay 
constraints of specific applications while minimizing the energy consumption.

\section{Architecture of mobile multimedia sensor networks}

Due to node mobility in MMSNs, some multimedia sensor nodes can move to various critical locations for collecting comprehensive information such as image or video stream. Previously, the issue of guaranteeing soft QoS delay for delivering multimedia streams while prolonging lifetime over a bandwidth-limited and unreliable sensor network is addressed by exploiting multiple node-disjointed paths, in order to achieve load-balancing, reduction of path interference, enlarged bandwidth aggregation and fast packet delivery. However, those work are targeted at multimedia transmission over static WSNs [1]. In comparison, the proposed MMSNs have the following features:

- Traditional WSNs have the intrinsic characteristic of scalar data collection (e.g., temperature, humidity, air pressure, etc.), which is hard to elaborate some complicated events and phenomena. In MMSNs, multimedia sensor nodes can provide more comprehensive information such as pictures, text message, audio or videos.

- The merging of mobility into multimedia sensor nodes further improve the network performance, such as locating mobile nodes to an optimal positions for fast multimedia services, approaching targets for enhanced event description with highresolution image or video streams, the additional capability for exploring a larger area of sensor nodes to disseminate multimedia streams, as well as various advantages in traditional mobile sensor networks (e.g., load balancing, energy efficiency, improving fairness on the data collection, and coverage optimization, etc.)

- Though the mobility of multimedia sensor node provides the advantage, the network topology becomes dynamic, which brings difficulties in both the data communication and data management.

Figure 1 shows a simple illustrative architecture of MMSN. When a mobile multimedia sensor node (MMN) moves in MMSNs, it periodically sends a multimedia flow at a new location. If a geographic routing scheme is used, the MMN sets up an individual path to the sink node for each multimedia flow. As time goes on, a series of paths will be built up while the MMN moves along a certain trajectory. Given the illustrative scenario shown in Figure 1, the sequence of the constructed paths to transmit multimedia traffic to the sink could be: Path-A, Path-B, Path-C, Path-D, Path-E. If the mobility mode and multimedia collections are controlled by other systems intelligently, more and more automated applications can be generated for industry and daily life.

\section{Illustrative application for MMSNs}

Figure 2 is an illustrative application of enabling location-aware mobile multimedia services for healthcare. In this application, Tom is an old person and needs care. He owns a smart house, where three RFID readers are deployed at the proximity of the three entrances to his house. A certain number of sensor nodes are deployed in his house to detect environmental parameters. In addition, there is a mobile multimedia sensor node,

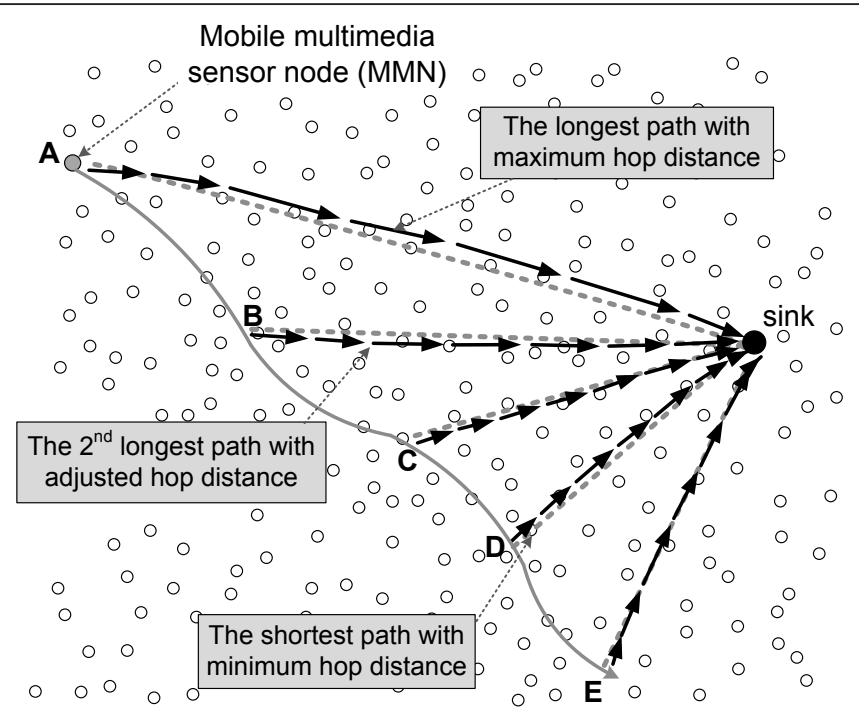

Figure 1 A simple illustrative architecture of mobile multimedia sensor network. 


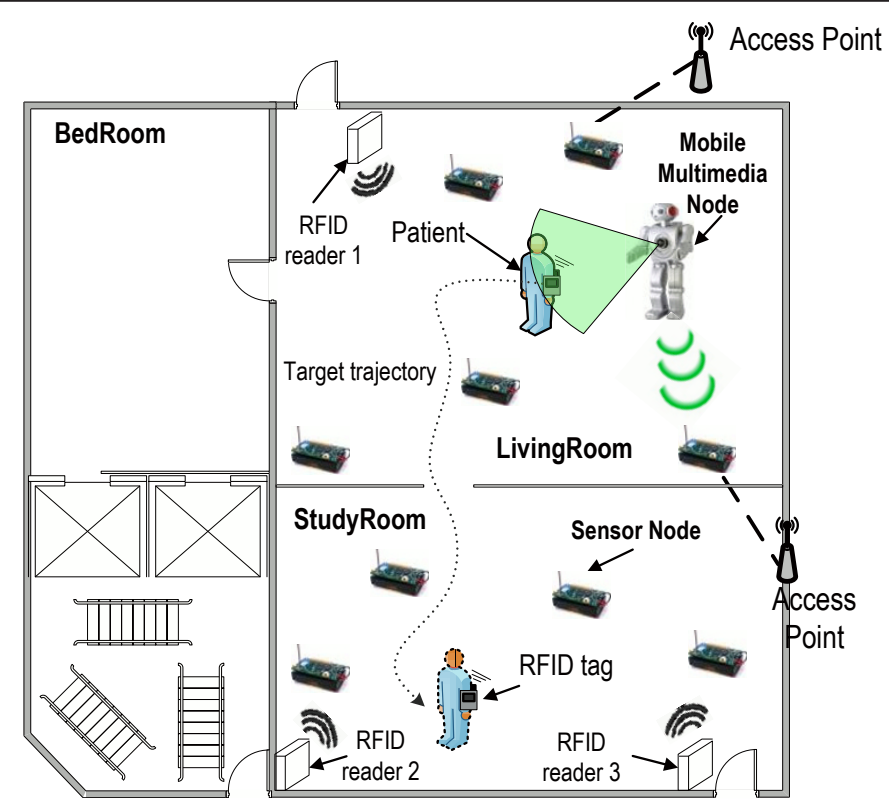

Figure 2 Illustrative application for MMSNs.

which is equipped with camera, as shown in Figure 2. To save energy, mobile node is powered off if no tasks are detected.

Once Tom enters his house through one of the entrances, his ID information stored in his tag will be transmitted to the nearby RFID reader. With the awareness of Tom's ID, the mobile node is activated. The system will periodically collect the three RSSI (received signal strength indication) values from the three RFID readers (i.e., RFID reader 1 , RFID reader 1 , and RFID reader 3 in Figure 2) to estimate the location of Tom. Assume the result of RFID-locating is living room, the mobile node will move to living room to take video for Tom. Due to his requirements for patient care, every details of his activities in specific rooms (i.e., living room and study room) need to be video-recorded. The video streaming is forwarded to the local video server through the access point. These video images are timestamped and stored in a directory associated with Tom's profile. When Tom moves from living room to study room, the result of RFID-locating will be changed to study room, and thus the mobile node will follow Tom to study room.

In this system, the vital signals of Tom are collected by body sensors. These body signals are subsequently updated into the database through sensor node(s) and/ or access point. Any abnormalities that do not require immediate treatment may be logged into the database and registered by Tom's RFID tag for future reference. Based on these body signals, a diagnosis might indicate more complicated multimedia information is needed to further ensure the accuracy of the diagnosis. On the other hand, the resolution of the camera in the mobile node can be adaptively adjusted by the severity of diagnosis result according to the contextual information (e. g., Tom's profile, behaviors, body signals, and environmental parameters, etc.). It might be possible for the doctor to remotely diagnose Tom immediately through the real-time video communications through the mobile node, as well as the physiological data information retrieved by a wireless body area network hosted by Tom. It's critical for the mobile node to approach to the object in a timely and energy-efficient fashion.

\section{Mobile multimedia geographic routing}

Since our design goal is to effectively support the multimedia service in MMSNs, we consider the performance in terms of both delay and energy. First, the delay guaranteeing is treated as the goal with top priority for the QoS provisioning. Then, the energy consumption should be minimized to enlarge the life time of sensors. This motivates to exploit the energy-delay trade-offs for the design of mobile multimedia geographic routing (MGR) scheme.

\subsection{Analysis of delay-energy trade-offs 5.1.1 Analysis of one-hop delay}

In this section, we analyze the latency between two neighboring nodes, which is the summation over the queuing, processing, propagation, and transmission delays: 
- Queuing delay: For the sake of simplicity, we assume a stable packet rate in our network. Then, queuing delay is considered to be a constant for each hop, which is denoted by $T_{q}$.

- Processing delay: With respect to processing delay, we assume that each node incurs similar delay to process and forward one packet with constant length. The processing delay is denoted by $T_{p}$.

- Propagation delay: This parameter can be neglected when compared to the other delays.

- Transmission delay: We assume that the size of a data packet does not change between a source-sink pair, its transmission delay (denoted by $T_{\mathrm{tx}}$ ) remains constant between any pair of intermediate sensor nodes.

Therefore, the delays taking place between any pair of intermediate nodes are considered to be similar in this paper, which can be estimated simply by $T_{\text {hop }}=T_{q}+T_{P}$ $+T_{\mathrm{tx}}$. Consequently, the delay between current node to the sink node is proportional to the hop count between the two nodes.

\subsubsection{The end-to-end energy consumption}

Given a constant packet size and a fixed propagation distance, we consider every sensor node will consume the same energy to forward the packet. Therefore, the end-to-end energy consumption for delivering a data packet from the source node to the sink node is proportional to the number of transmissions, i.e., the hop count. The basic energy model of one hop transmission in this paper is:

$$
E_{\mathrm{hop}}=C \cdot D_{\mathrm{hop}}^{\alpha}
$$

where $C$ is a constant value, $D_{\text {hop }}$ is the transmission distance, and the parameter $\alpha$ is the path loss exponent, depending on the environment, typically is equal to 2 when free space propagation is assumed. For the sake of simplicity, $C$ is set to 1 , and $\alpha$ is set to 2 . Then, $E_{\text {hop }}=D_{\text {hop }}^{2}$. Let $H_{s \rightarrow t}$ be the hop count from the source node to the sink node. Then, the end-to-end energy consumption can be estimated by:

$$
\begin{aligned}
E_{\text {ete }} & =\sum_{i=1}^{H_{s \rightarrow t}} E_{\text {hop }}(i) \\
& =E_{\mathrm{hop}} \cdot H_{s \rightarrow t} \\
& =D_{\text {hop }}^{2} \cdot H_{s \rightarrow t}
\end{aligned}
$$

which increases linearly with the value of $D_{\text {hop }}$. Motivated by an interesting feature that some sensor devices can transmit at different power levels [27], this paper assumes that the sensor node has the capability of power control to reduce end-to-end energy consumption.

\subsubsection{Energy-delay trade-off}

Typically, a geographic routing mechanism (e.g., GPSR [19]) intends to maximize packet progress at each hop in a greedy fashion. Since such a distance-based scheme introduces nearly maximal hop distance, the end-to-end delay could be minimized while more energy will be consumed based on our energy model.

However, achieving minimum delay is not beneficial for some delay sensitive applications when the minimum delay is smaller than the application-specific QoS delay boundary (i.e., $T_{\mathrm{QoS}}$ ). In the case that the earlier arrival of a data packets is not necessary, an intermediate sensor node can reduce the transmission power with a smaller transmission range for delivering packet to next hop in order to reduce energy consumption, but not too small to still be able to guarantee the delay objective.

\subsection{End-to-end delay objective}

Let $D_{s}^{t}$ denote the distance between source and sink. Let $R_{\max }$ denote the maximum transmission range of a sensor node. Then, the minimum end-to-end delay is equal to $T_{\min }=\frac{D_{s}^{t}}{R_{\max }}$, which is realized by the use of the shortest path with maximum progress at each hop. Then, for a certain network topology, an multimedia application is allowed to adjust application-specific endto-end delay $T_{\mathrm{QoS}}$ subject to the following constraint at least: $T$ Qos $>T_{\min }$, otherwise the QoS delay cannot be achieved.

\subsection{Calculating the desired hop distance at current node} Let $t_{s \rightarrow h}$ denote data packet's experienced delay up to current node. Let $t_{\text {current }}$ denote the current time when the routing decision is being made; let $t_{\text {create }}$ denote the time when the packet is created at the source node. Then, $t_{s \rightarrow h}$ can be easily calculated by the difference between $t_{\text {current }}$ and $t_{\text {create. }}$ Then, the reserved time credit for the data delivery from current node to the sink node, $T_{h \rightarrow t}$, can be calculated by:

$$
T_{h \rightarrow t}=T_{\mathrm{QoS}}-t_{s \rightarrow h}
$$

Based on $T_{h \rightarrow t}$ and $\mathrm{T}_{\text {hop }}$, the desired hop count from current node to the sink node can be estimated as

$$
H_{h \rightarrow t}=\frac{T_{h \rightarrow t}}{T_{\mathrm{hop}}}
$$

Upon the reception of data packet from its previous hop, the current node will know the position of the sink node. Then, distance from current node to the sink node, $D_{h \rightarrow t}$, can be calculated according to the positions of itself and the sink node. Let $D_{\text {hop }}$ denote the desired hop distance for next-hop-selection. 
Then,

$$
D_{\text {hop }}=\frac{D_{h \rightarrow t}}{H_{h \rightarrow t}}
$$

\subsection{Strategic location for next-hop-selection}

In this paper, strategic location means the ideal location of current node's next hop. Based on $D_{\text {hop }}$ calculated in Section 5.3, the strategic location of MGR is decided as in Figure 3

The absolute coordinates of the strategic location and a next hop candidate $j$ are denoted by $\left(x_{s}, y_{s}\right)$ and $\left(x_{j}, y_{j}\right)$, respectively. Then, the distance between $j$ and the strategic location (denoted by $\Delta D_{j}$ ) can be calculated by

$$
\Delta D_{j}=\sqrt{\left(x_{s}-x_{j}\right)^{2}+\left(y_{s}-y_{j}\right)^{2}}
$$

\subsection{Next-hop-selection in MGR}

A node receiving a data packet will calculate the coordinates of its strategic location. Then, MGR will select as the next hop node whose distance is closest to the strategic location, instead of the neighbor closest to the sink as in traditional geographical routing protocols. The pseudo-code of the next-hop-selection algorithm for MGR is shown in Table 2.

\section{Performance evaluation}

We implement our protocols and perform simulations using OPNET Modeler [28]. The network with 2,000 nodes is randomly deployed over a 2,000 $\mathrm{m} \times 1,000 \mathrm{~m}$ field. We let the sink node stay at a corner of the field and one MMN be located at the other corner. When simulation starts, the MMN will move back and forth along the diagonal line of the network field. We assume the sink node and the ordinary sensor nodes are stationary. Our sensor node implementation has a four-layer
Table 2 MGR-NextHop(POS $\left.{ }_{h}, \mathrm{POS}_{t}, T_{\text {Qos, }} T_{\text {hop }}\right)$ : Pseudo-code for selecting the neighbor with the minimum $D_{j}$ as

\section{NextHop}

begin

Notation

$h$ is the current node to select the next hop node;

$V_{h}$ is the set of node $h^{\prime} s$ neighbors in the forwarding area;

POS $_{h}$ is position of the current node;

$\mathrm{POS}_{t}$ is position of the sink node;

\section{initialization}

calculate $T_{h \rightarrow t}$ based on $T_{\text {Qos }}$ and $t_{s \rightarrow h i}$

calculate $H_{h \rightarrow t}$ based on $T_{h \rightarrow t}$ and $T_{\text {hopi }}$

calculate $D_{h} \rightarrow t$ based on $\mathrm{POS}_{h}$ and $\mathrm{POS}_{t i}$

calculate $D_{\text {hop }}$ based on $D_{h \rightarrow t}$ and $H_{h \rightarrow t i}$

for each neighbor $j$ in $V_{h}$ do

calculate $\Delta D_{j}$ according to Equation (5);

end for

for each neighbor $j$ in $V_{h}$ do

if $\Delta D_{j}=\min \left\{\Delta D_{k} \| k \in V_{h}\right\}$ then

select $j$ as NextHop;

break;

end if

end for

Return $j_{i}$

protocol structure. The sensor application module consists of a constant-bit-rate source, which generates delay sensitive multimedia traffic with a certain QoS requirements. We use IEEE 802.11 DCF as the underlying $\mathrm{MAC}$, and the maximum radio transmission range $\left(R_{\max }\right)$ is set to $60 \mathrm{~m}$.

We mainly consider the following four performance metrics:

- End-to-end Packet Delay: It includes all possible delays during data dissemination, caused by queuing,

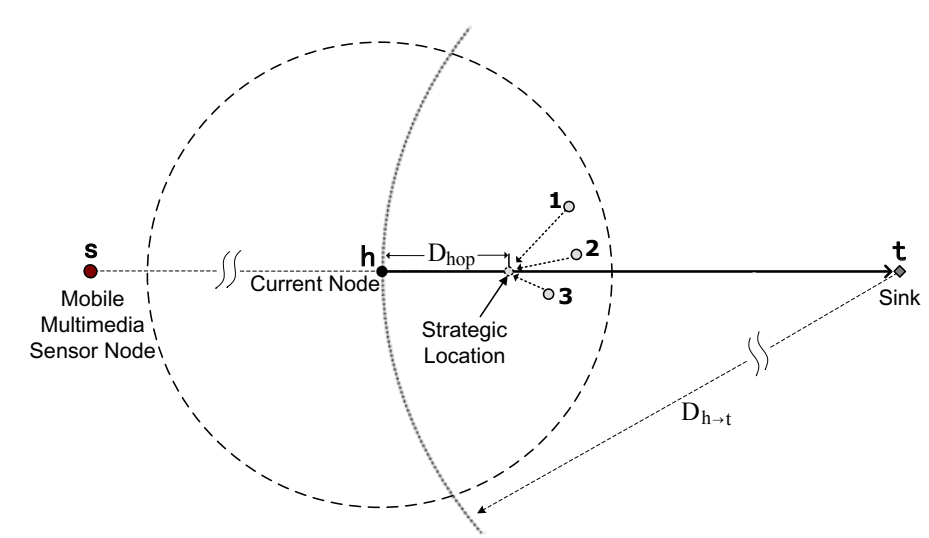

Figure 3 Illustration of the strategic location selection in MGR scheme. 


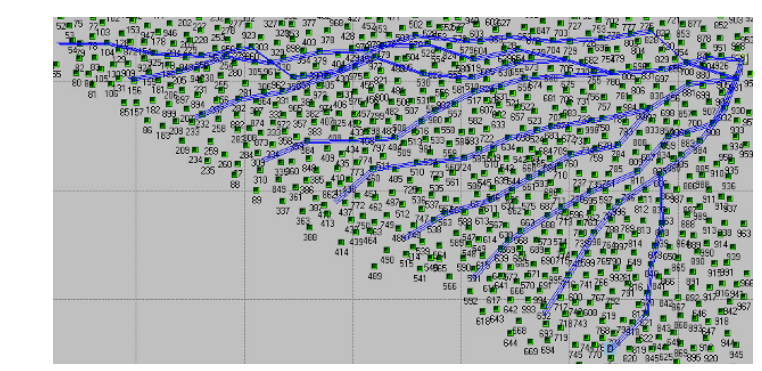

(a) GPSR

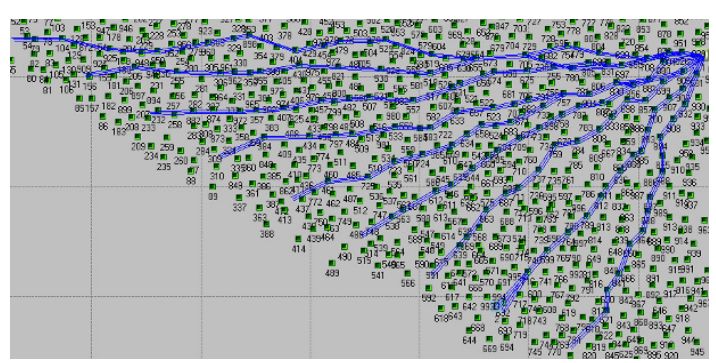

(b) MGR

Figure 4 Simulation animation with different routing schemes: (a) GRSR, (b) MGR

retransmission due to collision at the MAC, and transmission time.

- Energy Consumption: the energy consumption for a successful data delivery, which is calculated according to Equation (1).

- Average Energy Consumption: it is a running mean of ordinate values of input statistic, which is obtained by the statistics collection mode of "Average Filter" in OPNET simulation [28].

- Lifetime: It's the time when the first node exhausts its energy.

First, the proposed MGR scheme is compared to a pure shortest path based routing scheme (i.e., GRSR [19]). Figure 4 shows the snapshots of two OPNET simulations. The snapshots are for the path constructions when MMN moves along the diagonal line in the scenarios of GPSR and MGR, respectively. By comparison, the paths constructed by MGR are more straight. As shown in Figure 5a, the path lengths in GPSR and MGR are similar. However, MGR's hop distance is adaptively adjusted to save energy while keeping the end-to- end objective delay. Thus, the hop count in MGR is larger than pure distance-based routing scheme, as shown in Figure 5b.

The delay requirement $T_{\mathrm{QoS}}$ is set to $0.035 \mathrm{~s}$. As show in Figure 6a, both GPSR and MGR guarantee the QoS delay in most cases. In GPSR, paths have various delays ranging from 0.014 to $0.035 \mathrm{~s}$. By comparison, most of the delays in MGR change from 0.025 to $0.035 \mathrm{~s}$. The delay fluctuation of GPSR is much larger than MGR. It is because the GPSR does not have delay control mechanism without the consideration of MMN's up-todated location when it moves in the network.

As shown in Figure 6b, the energy consumption of GPSR is higher than that of MGR. It is because the maximum transmission range is always used by a greedy approach in GPSR. By comparison, in MGR, the end-toend delay is softly guaranteed while the energy is still saved. Figure 6c shows the comparison of average energy consumption. MGR saves about 30\% energy consumption when compared to GPSR. In our experiments, the simulation time corresponding to the last data point is also equivalent to the lifetime. As shown in Figure 6,

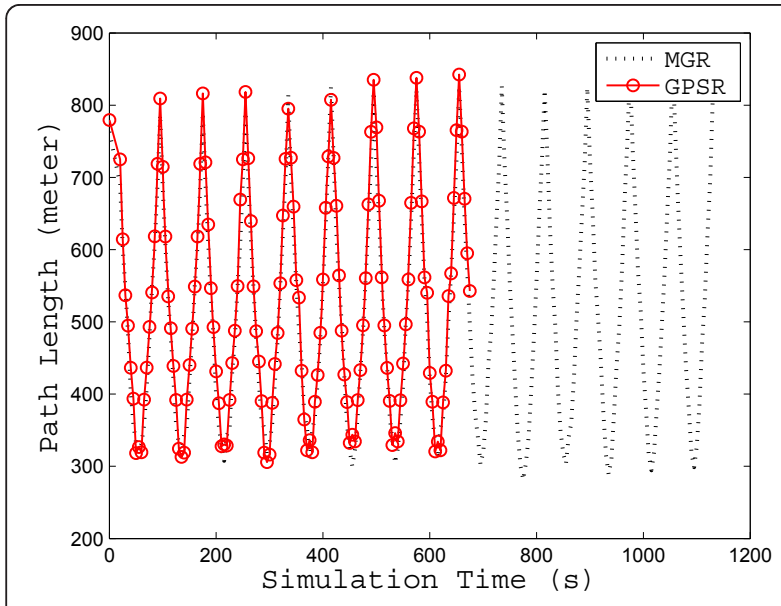

(a) Path Length

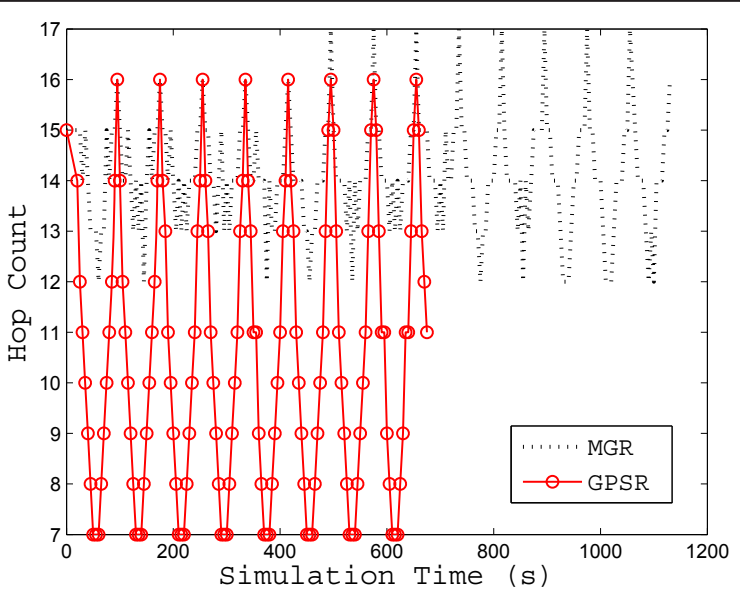

(b) Hop Count

Figure 5 Performance comparison: (a) path length, (b) hop count 


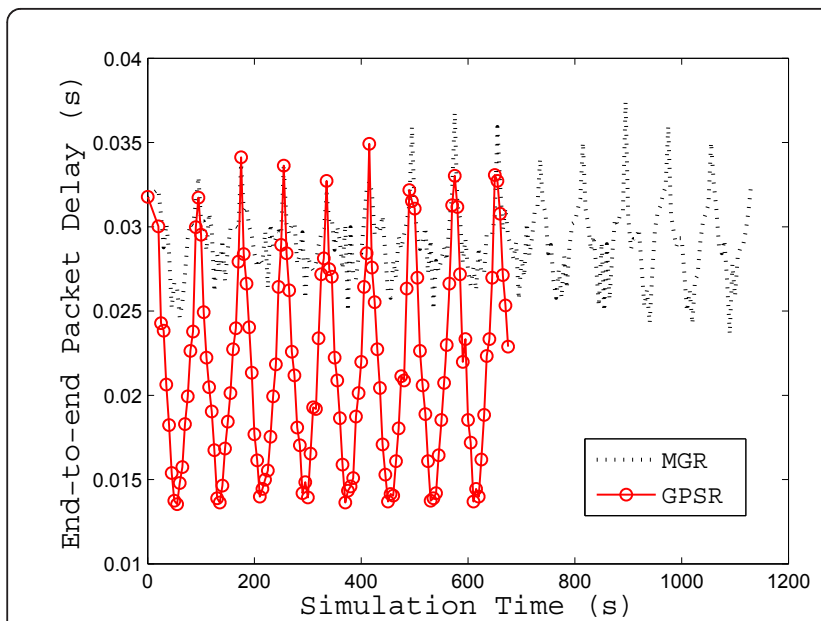

(a) End-to-end Packet Delay

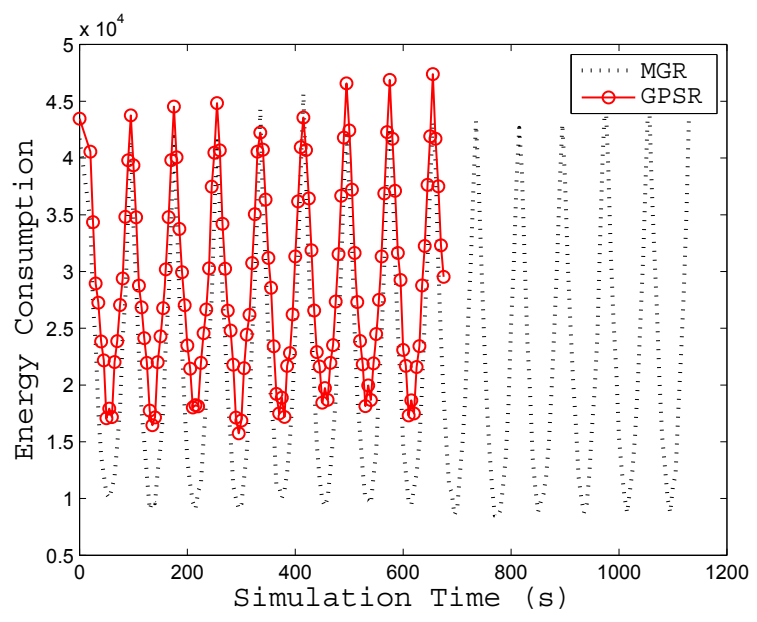

(b) Energy Consumption

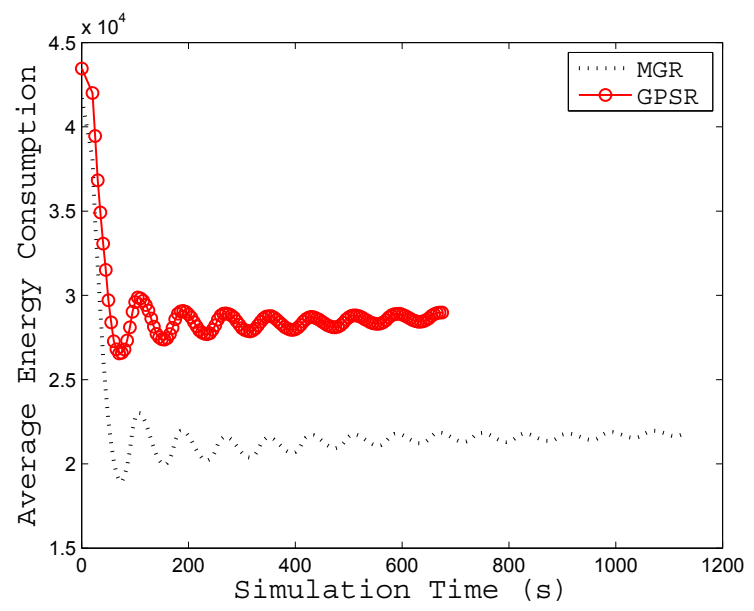

(c) Average Energy Consumption

Figure 6 Performance comparison: (a) end-to-end packet delay, (b) energy consumption, (c) average energy consumption.

the lifetimes of GPSR and MGR are 675 and 1,130 s, respectively, and MGR yields $455 \mathrm{~s}$ more lifetime than GPSR.

\section{Conclusion}

In this paper, we propose mobile multimedia sensor networks (MMSNs) where mobile multimedia sensor node (MMN) is exploited to enhance the sensor network's capability for event description. Then, the trade-offs of end-to-end delay and energy consumption for supporting multimedia service with delay QoS requirement are discussed. By utilizing location information, we design a routing algorithm named mobile multimedia geographic routing (MGR) for QoS provisioning in MMSNs. When MMN moves in the network, MGR is designed to minimize energy consumption and satisfy constraints on the average end-to-end delay of specific applications. The experiment results show the efficiency of MGR in satisfying QoS requirement while saving energy. In future, we will further improve MGR for more reliable and efficient QoS-oriented transmission scheme and adapt MGR for the scenarios with multiple multimedia flows per source-sink pair.

\section{Acknowledgements}

This research was supported by the IT R\&D program of KCA (10913-05004: Study on Architecture of Future Internet to Support Mobile Environments and Network Diversity).

\section{Author details}

${ }^{1}$ School of Computer Science and Engineering, Seoul National University, Seoul, Korea ${ }^{2}$ Institute of Computer Science and Information Engineering, National Ilan University, Ilan, Taiwan ${ }^{3}$ Department of Electrical and Computer Engineering, University of Massachusetts, Boston, MA, USA

\section{Competing interests}

The authors declare that they have no competing interests. 
Received: 29 June 2011 Accepted: 7 November 2011

Published: 7 November 2011

\section{References}

1. I Akyildiz, T Melodia, K Chowdhury, A survey on wireless multimedia sensor networks. Comput Netw. 51(4), 921-960 (2007). doi:10.1016/j. comnet.2006.10.002

2. M Chen, S Gonzalez, Q Zhang, VCM Leung, Code-centric RFID system based on software agent intelligence. IEEE Intell Syst. 25(2), 12-19 (2010)

3. M Chen, S Gonzalez, H Cao, Y Zhang, S Vuong, Enabling low bit-rate and reliable video surveillance over practical wireless sensor networks. J Supercomput (2010)

4. M Chen, V Leung, S Mao, Y Yuan, Directional geographical routing for realtime video communications in wireless sensor networks. J Comput Commun. 30(17), 3368-3383 (2007). doi:10.1016/j.comcom.2007.01.016

5. L Shu, M Chen, Multimedia over Sensor Networks. IEEE COMSOC MMTC ELetter. 5(5), 59-61 (2010)

6. M Chen, S Gonzalez, A Vasilakos, H Cao, VCM Leung, Body area networks: a survey. ACM Mob Netw Appl. 16(2), 171-193 (2011). doi:10.1007/s11036010-0260-8

7. M Chen, J Guan, H Liu, Enabling fast brain-computer interaction by singletrial extraction of visual evoked potentials. J Med Syst

8. M Chen, S Gonzalez, VCM Leung, Applications and design issues of mobile agents in wireless sensor networks. IEEE Wirel Commun. 14(6), 20-26 (2007)

9. Min Chen, L Yang, T Kwon, L Zhou, M Jo, Itinerary planning for energyefficient agent communication in wireless sensor networks. IEEE Trans Veh Technol. 60(7), 3290-3299 (2011)

10. M Chen, T Kwon, Y Yuan, Y Choi, VCM Leung, Mobile agent-based directed diffusion in wireless sensor networks. EURASIP J Appl Signal Process. 2007(1), 219-242 (2007)

11. M Chen, T Kwon, Y Yuan, V Leung, MAWSN: Mobile agent based wireless sensor networks. J Comput. 1(1), 14-21 (2006)

12. C-X Wang, M Pätzold, Q Yao, Stochastic modeling and simulation of frequency correlated wideband fading channels. IEEE Trans Veh Techno. 56(3), 1050-1063 (2007)

13. C-X Wang, $X$ Hong, HH Chen, JS Thompson, On capacity of cognitive radio networks with average interference power constraints. IEEE Trans Wirel Commun. 8(4), 1620-1625 (2009)

14. X Ge, K Huang, C-X Wang, X Hong, X Yang, Capacity analysis of a multi-cell multi-antenna cooperative cellular network with co-channel interference. IEEE Trans Wirel Commun. 10(10), 3298-3309 (2011)

15. C-X Wang, X Hong, X Ge, X Cheng, G Zhang, J Thompson, Cooperative MIMO channel models: a survey. IEEE Commun Mag. 48(2), 80-87 (2010)

16. O Ghica, G Trajcevski, P Scheuermann, N Valtchanov, Z Bischof, Controlled multi-path routing in sensor networks using bezier curves. Comput J (2010)

17. I Stojmenovic, Position-based routing in ad hoc networks. IEEE Commun Mag. 40(7), 128-134 (2002). doi:10.1109/MCOM.2002.1018018

18. P Bose, P Morin, I Stojmenovic, J Urrutia, Routing with guaranteed delivery in ad hoc wireless networks, in Proceedings of ACM DIAL 1999, Seattle, USA, pp. 48-55 (1999)

19. B Karp, HT Kung, GPSR: Greedy perimeter stateless routing for wireless networks, in Proceedings of ACM MobiCom 2000, Boston, Mass, USA, pp. 243-254 (2000)

20. M Popescu, E Florea, Linking clinical events in elderly to in-home monitoring sensor data: a brief review and a pilot study on predicting pulse pressure. J Comput Sci Eng. 2(2), 180-199 (2008)

21. S Raazi, S Lee, A survey on key management strategies for different applications of wireless sensor networks. J Comput Sci Eng. 4(1), 23-51 (2010)

22. Y Liu, W Zhang, K Akkaya, Static worst-case energy and lifetime estimation of wireless sensor networks. J Comput Sci Eng. 4(2), 128-152 (2010)

23. $T H e, J$ Stankovic, L Chenyang, $T$ Abdelzaher, SPEED a stateless protocol for real-time communication in sensor networks, in Proceedings of IEEE ICDCS'03, pp. 46-55 (2003)

24. E Felemban, C Lee, E Ekici, MMSPEED: multipath Multi-SPEED protocol for QOS guarantee of reliability and. Timeliness in wireless sensor networks. IEEE Trans Mob Comput. 5(6), 738-754 (2006)

25. Y Yuan, ZK Yang, ZH He, JH He, An integrated energy aware wireless transmission system for QoS provisioning in wireless sensor network. J Comput Commun. 29(2), 162-172 (2006). doi:10.1016/j.comcom.2005.05.015
26. M Chen, T Kwon, Y Choi, Energy-efficient differentiated directed diffusion (EDDD) for real-time traffic in wireless sensor networks. J Comput Commun. 29(2), 231-245 (2006). doi:10.1016/j.comcom.2005.05.019

27. M Chen, VC Leung, S Mao, Y Xiao, I Chlamtac, Hybrid geographic routing for flexible energy-delay tradeoff. IEEE Trans. Veh Technol. 58(9), 4976-4988 (2009)

28. OPNET Modeler, http://www.opnet.com

doi:10.1186/1687-1499-2011-159

Cite this article as: Chen et al.: Mobile multimedia sensor networks:

architecture and routing. EURASIP Journal on Wireless Communications and Networking 2011 2011:159.

\section{Submit your manuscript to a SpringerOpen ${ }^{\mathcal{O}}$ journal and benefit from:}

- Convenient online submission

- Rigorous peer review

- Immediate publication on acceptance

- Open access: articles freely available online

- High visibility within the field

- Retaining the copyright to your article

Submit your next manuscript at $>$ springeropen.com 